\title{
COVID-19 and Periodontal Disease
}

\section{Nikesh Moolya*}

Department of Periodontics, Yeshwantrao Chavan Memorial Medical and Rural

Foundation Dental College, India

*Corresponding Author: Nikesh Moolya, Department of Periodontics, Yeshwantrao

Chavan Memorial Medical and Rural Foundation Dental College, India.
Received: July 05, 2021

Published: September 01, 2021

(C) All rights are reserved by Nikesh Moolya.
Periodontal disease can cause gingival inflammation, bleeding from gums, bad breath, and if left untreated could lead to tooth mobility and tooth loss. The latest findings from the AAP and the CDC and Prevention have estimated that up to half of adults aged 30 years and above in US have some forms of periodontal problems. Since the last few years, numerous trials have shown the existence of a two-way relationships between periodontal disease and systemic conditions. There is also emerging evidence regarding interrelationship between COVID-19 and periodontal disease.

Coronavirus SARS-CoV-2 is a viral strain from a severe form of acute respiratory syndrome related coronavirus known as member of the Coronaviridae. Current concepts suggest that increase in release of interleukins from host cells called as the cytokine storm, has strong relationship with disease progression in patients with coronavirus disease 2019 (COVID-19). This respiratory tract infection has till now resulted in over 18 crore confirmed cases and almost 39 lakh deaths as of June $30^{\text {th }}, 2021$.

A prospective study carried out using the national electronic health record database in Qatar in 2020, analysed patient cases with severe COVID-19 complications (ICU admissions or assisted ventilation, death). The control group had COVID-19 patients without major complications. Periodontal parameters in the two groups were studied using dental $x$-rays from the same database. Periodontitis were at least three times more likely to experience COVID-19 complications in the 568 patients studied, including ICU admission, death and the need for ventilation. COVID-19 patients with periodontitis also showed increased levels of biomarkers associated with worsened disease outcomes including D-dimer, white blood cell levels, and C-reactive protein changes. These stud- ies reinforce the importance of adequate non-surgical therapy in COVID 19 patients aft.er recovery.

Every transmissible disease has always imposed further restrictions in infection control measures in periodontal practice. The majority of these diseases are closely related to the respiratory system. Various trials have postulated how periodontal aerosol generating procedures could lead to communication of contagious respiratory pathogens in dental operatories. Though development in research have led to the introduction of many new methods to prevent disease transmission, it is not possible to provide complete protection in the dental setting. Since the time SARS-CoV affected many patients, the Center for Disease Control and Prevention (CDC) and American Dental Association (ADA) have recommended the avoidance of any AGP in patients with active SARS. Patients with such a serious illness must not undergo any elective dental treatment. ADA has currently recommended carrying out only emergency dental treatment to limit the rapid spread of the deadly coronavirus. Periodontal diseases are preventable and noncontagious. Online patient education and motivation, diagnosis, and oral hygiene instructions (OHIs) have the major advantage as it is less time consuming with patients in the clinic, while proper practice of oral hygiene will decrease clinical signs and symptoms of gingival inflammation before visiting the periodontist. Periodontal clinics held virtually can help minimize the clinic interview duration. Short and simple periodontal examination is mandatory in such settings. One visit non-surgical periodontal therapy is better than multiple visits for treatment of patients with periodontitis as it involves minimum cross contamination. Preprocedural antibacterial and antiviral mouthwashes, as a rule is compulsory before periodontal procedures. Use of hand instrumentation and polishing cups is manda- 
tory. Frequent use of sterile gauze instead of irrigation and use of saliva ejectors can reduce incidence of cross contamination. N95 masks, face shields and surgical protective gowns should be worn throughout clinical procedures. Excellent surface decontamination and sufficient air ventilation procedures should be carried out. The dental unit waterlines have to be maintained on a regular basis and disinfected regularly. Sufficient time should be allowed for room decontamination and fumigation between patients.

\section{Volume 5 Issue 10 October 2021}

(C) All rights are reserved by Nikesh Moolya. 